\title{
Equivalent Martingale Measure in Asian Geometric Average Option Pricing
}

\author{
Yonggang Zhu \\ School of Science, China Three Gorges University, Yichang, China \\ Email: zygpf@163.com
}

Received 29 May 2014; revised 8 July 2014; accepted 27 July 2014

Copyright (C) 2014 by author and Scientific Research Publishing Inc.

This work is licensed under the Creative Commons Attribution International License (CC BY). http://creativecommons.org/licenses/by/4.0/

(c) (i) Open Access

\begin{abstract}
The general situation of the Black-Scholes Option Pricing Model was discussed under the assumption of the arbitrage-free market, and the pricing of Asian geometric average options with fixed strike price was analyzed at any valid time. Consequently, the price formula of the Asian geometric average options was drawn using the equivalent martingale measure and the significance of the study was also indicated.
\end{abstract}

\section{Keywords}

Asian Geometric Average Options, Equivalent Martingale Measure, Black-Scholes Option Pricing Model, Strike Price

\section{Introduction}

Asian option, also known as the average price of options, was one of the derivatives of the stock options, and was firstly introduced by the American Bankers Trust Company (Bankers Trust) in Tokyo, Japan, on the basis of the lessons learned from the option implementations, such as real options, virtual options and stock options. It was a kind of exotic options, which was the most active one in financial derivative market, with the difference of the limitation of the exercise price from the usual stock option, that is, its exercise price was the average secondary market price of the stock price implemented during the current six months.

In this paper, after the Black-Scholes [1] Option Pricing Model was fully understood, the pricing of Asian options was discussed: It was assumed that the underlying asset price was driven by the geometric Brownian motion, that is, lognormal distribution. By using the random variables with the same Second moment driven by the lognormal distribution to approximate the arithmetic average of the underlying asset price, the approximate solution of the arithmetic average price of Asian put and call option with fixed exercise price was obtained, and the application of the equivalent martingale measure in the pricing of financial derivatives was further expanded 


\section{$[2]$.}

\section{Model and Formulas}

Generally, the stock market could be described as a probability space with a $\sigma$-stream, that is, $(\Omega, \mathcal{F}, P)$; It was supposed that the market could meet the following conditions:

(1) The market was an efficient frictionless market including two assets: one was the risk-free assets, known as the bonds, whose price process was denoted by $B_{t},(t>0)$; another was the risky assets, called stocks, the price process was denoted by $S_{t},(t>0)$. They satisfied the following formula separately:

$$
\begin{aligned}
& \mathrm{d} S_{t}=\mu S_{t} \mathrm{~d} t+\sigma S_{t} \mathrm{~d} W_{t}^{P}, \quad 0 \leq t \leq T \\
& B_{t}^{-1} \mathrm{~d} B_{t}=r \mathrm{~d} t, \quad 0 \leq t \leq T
\end{aligned}
$$

where, $\mu$ denotes the expectation of the yield rate, $\sigma$ denotes firm-value process volatility, $T$ denotes time to expiration of option, $r$ denotes the risk-free interest rate and they all are constants. $d W_{t}^{P}$ denotes the instantaneous increment of the Brownian motion under the probability measurer $P$ at time $t$;

(2) Security trading is continuous and there are no transactions costs or taxes;

(3) There are no dividends to be payoff during options being held.

Definition 1 Let $(\Omega, \mathcal{F}, P)$ be a probability space and $\mathcal{F}_{0} \subset \mathcal{F}_{1} \subset \cdots \subset \mathcal{F}_{n}$ be an increasing chain of $\sigma$ fields spanning $\mathcal{F}$, which $\mathcal{F}_{0}=\{\Phi, \Omega\}$ and $\left(W_{t}^{P}, 0 \leq t \leq T\right)$ be a standard Brownian motion. $\mathcal{F}_{t}$ be a $\sigma$ algebra of spanning $W_{t}^{P}$.

Let measures $Q$ satisfy:

$$
\frac{\mathrm{d} Q}{\mathrm{~d} P}=\exp \left[\int_{0}^{t} \frac{r-\mu}{\sigma} \mathrm{d} W_{s}^{P}-\frac{1}{2} \int_{0}^{t}\left(\frac{r-\mu}{\sigma}\right)^{2} \mathrm{~d} s\right]=\exp \left[\frac{r-\mu}{\sigma} \Delta W_{t}^{P}-\frac{1}{2}\left(\frac{r-\mu}{\sigma}\right)^{2} t\right]
$$

and set $Z_{t}=\frac{\mathrm{d} Q}{\mathrm{~d} P}$, we know that $Z_{T}$ is a martingale since $E^{P} \exp \left[\frac{1}{2} \int_{0}^{t}\left(\frac{r-\mu}{\sigma}\right)^{2} \mathrm{~d} s\right]<\infty$. Then measure $Q$ is defined a martingale measure equivalent to measure $P$, where $E^{P}(\cdot)$ denotes the expectation of random variable under probability measure $P$ [3].

Lemma 1 The dynamics of the share price under probability measure $Q$ :

$$
S_{t}=S \exp \left[\left(r-\frac{1}{2} \sigma^{2}\right) t+\sigma \Delta W_{t}^{Q}\right]
$$

where $S$ denotes the share price now and $\Delta W_{t}^{Q}=W_{t}^{Q}-W_{0}^{Q}, \Delta W_{t}^{Q} \sim N^{Q}(0, t)$.

Prove Because the share price process satisfy the formula (1), using Ito's theorem, we have:

$$
\mathrm{d} \ln S_{t}=\left(r-\frac{\sigma^{2}}{2}\right) \mathrm{d} t+\sigma \mathrm{d} W_{t}^{P}, \quad 0 \leq t \leq T
$$

Then we get the dynamics of the share price under probability measure $P$ :

$$
S_{t}=S \exp \left[\left(r-\frac{1}{2} \sigma^{2}\right) t+\sigma \Delta W_{t}^{P}\right]
$$

where $\Delta W_{t}^{P}=W_{t}^{P}-W_{0}^{P}$ and $\Delta W_{t}^{P} \sim N^{P}(0, t)$.

Let $W_{t}^{P}=W_{t}^{Q}+\int_{0}^{t}\left(\frac{r-\mu}{\sigma}\right) \mathrm{d} s$, by definition 1 and Girsanov's theorem we get that the random process $\left(W_{t}^{Q}, 0 \leq t \leq T\right)$ is a Brownian motion on $(\Omega, \mathcal{F}, Q)$ and:

$$
\mathrm{d} W_{t}^{P}=\mathrm{d} W_{t}^{Q}+\left(\frac{r-\mu}{\sigma}\right) \mathrm{d} t
$$




$$
E\left(Z_{T} I_{A}\right)=P^{Q}(A), \forall A \subset F_{T}
$$

where $E(\bullet)$ denotes the expectation of random variable in probability measure $P$ and $P^{Q}(\bullet)$ denotes the probability of random variable in measure $P, I_{A}$ is an indicator function of set $A$. Substituting (5) into (3), we get:

$$
\mathrm{d} \ln S_{t}=\left(r-\frac{\sigma^{2}}{2}\right) \mathrm{d} t+\sigma \mathrm{d} W_{t}^{Q}, \quad 0 \leq t \leq T
$$

Thus we have that under probability measure $Q$ :

$$
S_{t}=S \exp \left[\left(r-\frac{1}{2} \sigma^{2}\right) t+\sigma \Delta W_{t}^{Q}\right] .
$$

The proof is completed.

Lemma 2 Let $X_{T}=\ln \frac{S_{T}}{S}, Y_{T}=\ln \frac{S_{T}}{S}, \mu=r-\frac{1}{2} \sigma^{2}$, then the distribution function of $\left(X_{T}, Y_{T}\right)$ is:

$$
P\left\{X_{T} \geq x, Y_{T} \geq y\right\}=N\left(\frac{-x+\mu T}{\sigma \sqrt{T}}\right)-\mathrm{e}^{\frac{2 \mu}{\sigma^{2}}} N\left(\frac{-x+\mu T+2 y}{\sigma \sqrt{T}}\right)
$$

Definition 2 Using a bond as the denominated unit, $B_{t}=\exp \left(-\int_{0}^{t} r(s) \mathrm{d} s\right)$ was the process of the discount factor, and $\tilde{S}_{t}=B(t) S(t)$ was the value process of discounted assets.

\section{Asian Geometric Average Options Pricing}

In general, for the contingent claim, the risk-neutral pricing principle [4] was obtained as followed.

Theorem 1 It was supposed that the market was arbitrage-free, so that the value of the process of any asset $V(t)$ at time $t$ was:

$$
V(t)=E^{Q}\left(V(T) \exp \left(-\int_{t}^{T} r(s) \mathrm{d} s\right) \mid F_{t}\right) .
$$

Considering one bearish Asian option, its return at expiration time was: $V_{p}(t)=\left(K-J_{T}\right)^{+}$, where,

$$
J_{T}=\exp \left(\frac{1}{T} \int_{0}^{T} \ln S_{\tau} \mathrm{d} \tau\right) .
$$

Under the conditions of arbitrage-free market and from Theorem 1, the price at time $t$ was:

$$
V_{p}(t)=E^{Q}\left(V_{p}(T) \exp \left(-\int_{t}^{T} r(s) \mathrm{d} s\right) \mid F_{t}\right)=\exp \left(-\int_{t}^{T} r(s) \mathrm{d} s\right) E^{Q}\left(\left(K-J_{T}\right)^{+} \mid F_{t}\right)
$$

To get the specific expression of $V_{p}(t)$, the key was to obtain the distribution of $J_{T}$ under the condition $F_{t}$. Made $Y=\frac{1}{T} \int_{0}^{T} \ln S_{\tau} \mathrm{d} \tau$, then $J_{T}=\mathrm{e}^{Y}$, and:

$$
\ln \tilde{S}(t)=\ln S_{0}-\frac{1}{2} \int_{0}^{t} \sigma^{2}(s) \mathrm{d} s+\int_{0}^{t} \sigma(s) \mathrm{d} \tilde{W}(s)
$$

Thereby,

$$
\ln S(t)=\ln S_{0}+\int_{0}^{t}\left(r(s)-\frac{1}{2} \sigma^{2}(s)\right) \mathrm{d} s+\int_{0}^{t} \sigma(s) \mathrm{d} \tilde{\mathrm{W}}(s)
$$

Therefore, 


$$
\begin{aligned}
Y= & \frac{1}{T} \int_{0}^{T} \ln S_{\tau} \mathrm{d} \tau=\frac{1}{T}\left(\int_{0}^{t} \ln S_{\tau} \mathrm{d} \tau+\int_{t}^{T} \ln S_{\tau} \mathrm{d} \tau\right) \\
= & \frac{1}{T}\left\{\int_{0}^{t} \ln S_{\tau} \mathrm{d} \tau+\int_{t}^{T}\left[\ln S_{0}+\int_{0}^{t}\left(r(s)-\frac{1}{2} \sigma^{2}(s)\right) \mathrm{d} s+\int_{0}^{t} \sigma(s) \mathrm{d} \tilde{W}(s)+\int_{t}^{\tau} \sigma(s) \mathrm{d} \tilde{W}(s)\right] \mathrm{d} \tau\right\} \\
= & \frac{1}{T} \int_{0}^{t} \ln S_{\tau} \mathrm{d} \tau+\frac{T-t}{T} \ln S_{0}+\frac{1}{T} \int_{t}^{T} \int_{0}^{\tau}\left(r(s)-\frac{1}{2} \sigma^{2}(s)\right) \mathrm{d} s \mathrm{~d} \tau \\
& +\frac{T-t}{T} \int_{0}^{t} \sigma(s) \mathrm{d} \tilde{W}(s)+\frac{1}{T} \int_{t}^{T} \int_{0}^{\tau} \sigma(s) \mathrm{d} \tilde{W}(s) \mathrm{d} \tau .
\end{aligned}
$$

Written as:

$$
\begin{gathered}
\tilde{\mu}_{t}=\frac{1}{T} \int_{0}^{t} \ln S_{\tau} \mathrm{d} \tau+\frac{T-t}{T} \ln S_{0}+\frac{1}{T} \int_{t}^{T} \int_{0}^{\tau}\left(r(s)-\frac{1}{2} \sigma^{2}(s)\right) \mathrm{d} s \mathrm{~d} \tau+\frac{T-t}{T} \int_{0}^{t} \sigma(s) \mathrm{d} \tilde{W}(s), \\
\tilde{\eta}_{t}=\frac{1}{T} \int_{t}^{T} \int_{0}^{\tau} \sigma(s) \mathrm{d} \tilde{W}(s) \mathrm{d} \tau,
\end{gathered}
$$

Then,

$$
J(t)=\mathrm{e}^{\tilde{\mu}_{t}+\tilde{\eta}_{t}}
$$

Theorem $2 \tilde{\eta}_{t}=\frac{1}{T} \int_{t}^{T} \int_{0}^{\tau} \sigma(s) \mathrm{d} \tilde{\mathrm{W}}(s) \mathrm{d} \tau$ was driven by the normal distribution $N\left(0, \tilde{\sigma}_{t}^{2}\right)$,

where, $\tilde{\sigma}_{t}^{2}=\frac{1}{T^{2}} \int_{t}^{T}(T-s)^{2} \sigma^{2}(s) \mathrm{d} s$.

Note: It could be deduced from the Lemma 1.

Theorem 3 It was supposed that the market was arbitrage-free, then the price of Asian put options $V_{p}(t)=\left(K-J_{T}\right)^{+}$at any valid time $t$ was:

$$
V_{p}(t)=\mathrm{e}^{-\int_{t}^{T} r(s) \mathrm{d} s}\left[K N\left(\frac{\ln K-\tilde{\mu}_{t}}{\tilde{\sigma}_{t}}\right)-\mathrm{e}^{\tilde{\mu}_{t}+\frac{1}{2} \tilde{\sigma}_{t}^{2}} N\left(\frac{\ln K-\tilde{\mu}_{t}}{\tilde{\sigma}_{t}}\right)\right]
$$

where, $N(t)=\int_{-\infty}^{t} \frac{1}{\sqrt{2 \pi}} \mathrm{e}^{-\frac{x^{2}}{2}} \mathrm{~d} x$.

\section{Prove}

$$
\begin{aligned}
V_{p}(t) & =\mathrm{e}^{-\int_{t}^{T} r(s) \mathrm{ds}}\left[K N\left(\frac{\ln K-\tilde{\mu}_{t}}{\tilde{\sigma}_{t}}\right)-\mathrm{e}^{\tilde{\mu}_{t}+\frac{1}{2} \tilde{\sigma}_{t}^{2}} N\left(\frac{\ln K-\tilde{\mu}_{t}}{\tilde{\sigma}_{t}}\right)\right] \\
& =\mathrm{e}^{-\int_{t}^{T} r(s) \mathrm{ds}} E^{Q}\left(\left(K-\mathrm{e}^{\tilde{\mu}_{t}+\tilde{\eta}_{t}}\right)^{+} \mid F_{t}\right) \\
& =\left.\mathrm{e}^{-\int_{t}^{T} r(s) \mathrm{ds}} E^{Q}\left(\left(K-\mathrm{e}^{x+\tilde{\eta}_{t}}\right)^{+} \mid F_{t}\right)\right|_{x=\tilde{\mu}_{t}} \\
& =\mathrm{e}^{-\int_{t}^{T} r(s) \mathrm{ds} s} \int_{-\infty}^{\ln K-\tilde{\mu}_{t}}\left(K-\mathrm{e}^{\tilde{\mu}_{t}+y}\right) \frac{1}{\sqrt{2 \pi} \tilde{\sigma}_{t}} \mathrm{e}^{-\frac{y^{2}}{2 \tilde{\sigma}_{t}^{2}}} \mathrm{~d} y \\
& =\mathrm{e}^{-\int_{t}^{T} r(s) \mathrm{ds} s}\left[K N\left(\frac{\ln K-\tilde{\mu}_{t}}{\tilde{\sigma}_{t}}\right)-\mathrm{e}^{\tilde{\mu}_{t}+\frac{1}{2} \tilde{\sigma}_{t}^{2}} N\left(\frac{\ln K-\tilde{\mu}_{t}}{\tilde{\sigma}_{t}}\right)\right] .
\end{aligned}
$$

The proof was completed.

Similarly, the price of Asian call options $V_{c}(T)=\left(J_{T}-K\right)^{+}$at any time $t$ could be obtained: 


$$
V_{c}(t)=\mathrm{e}^{-\int_{t}^{T} r(s) \mathrm{d} s}\left[\mathrm{e}^{\tilde{\mu}_{t}+\frac{1}{2} \tilde{\sigma}_{t}^{2}} N\left(\frac{\ln K-\tilde{\mu}_{t}-\tilde{\sigma}_{t}}{\tilde{\sigma}_{t}}\right)-K N\left(-\frac{\ln K-\tilde{\mu}_{t}}{\tilde{\sigma}_{t}}\right)\right]
$$

where, $N(t)=\int_{-\infty}^{t} \frac{1}{\sqrt{2 \pi}} \mathrm{e}^{-\frac{x^{2}}{2}} \mathrm{~d} x$.

Deduction 1 For Asian geometric average options, the parity relationship between call option and put option was:

$$
V_{c}(t)-V_{p}(t)=\mathrm{e}^{-\int_{t}^{T} r(s) \mathrm{d} s}\left[\mathrm{e}^{\tilde{\mu}_{t}+\frac{1}{2} \tilde{\sigma}_{t}^{2}}-K\right] .
$$

Deduction 2 When the interest rate $r$ and the volatility of stock returns $\sigma^{2}$ were constant, there was:

$$
\tilde{\mu}_{0}=\ln S_{0}+\frac{T}{2}\left(r-\frac{1}{2} \sigma^{2}\right), \quad \tilde{\sigma}_{0}^{2}=\ln S_{0}+\frac{T}{2}\left(r-\frac{1}{2} \sigma^{2}\right)=\frac{T}{3} \sigma^{2} .
$$

\section{Acknowledgements}

Natural Research Foundation of Education Bureau of Hubei Province of China, NO. 20101202, Yichang Municipal Science and Technology Planning Project, NO. A2010-302-18.

\section{References}

[1] Black, F. and Scholes, M. (1973) The Pricing of Options Corporate Liabilities. Journal of Political Economy, 81, 637659. http://dx.doi.org/10.1086/260062

[2] Zhu Y.G. (2009) Applications of Equivalent Martingales Model in Pricing Warrants. Proceedings of Conference on International Institute of Applied Statistics Studies, Recent Advance in Statistic Application and Related Areas, Sydney, 209-212.

[3] Kong, F.L. (1998) Large Number Laws for Banach Space-Valued Amart. Acta Mathematica Sinica, 41, 667-672.

[4] Martin, B. (1997) Finacial Calculus. Cambridge University Press, Cambridge. 
Scientific Research Publishing (SCIRP) is one of the largest Open Access journal publishers. It is currently publishing more than 200 open access, online, peer-reviewed journals covering a wide range of academic disciplines. SCIRP serves the worldwide academic communities and contributes to the progress and application of science with its publication.

Other selected journals from SCIRP are listed as below. Submit your manuscript to us via either submit@scirp.org or Online Submission Portal.
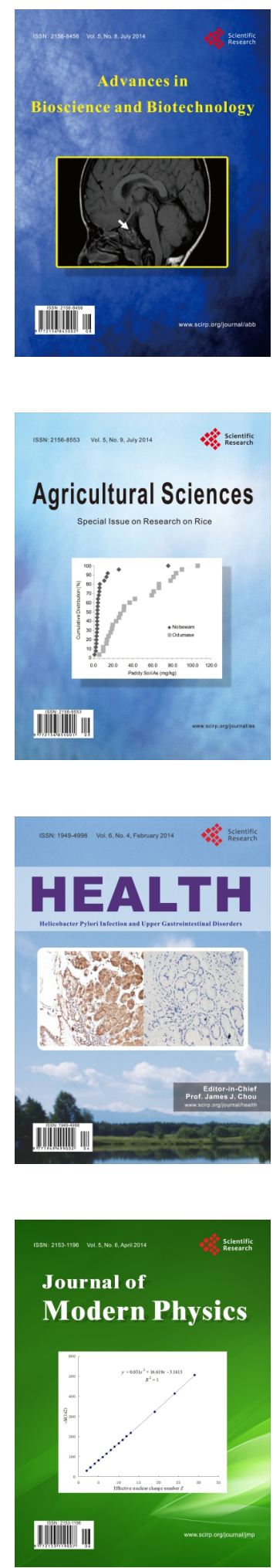
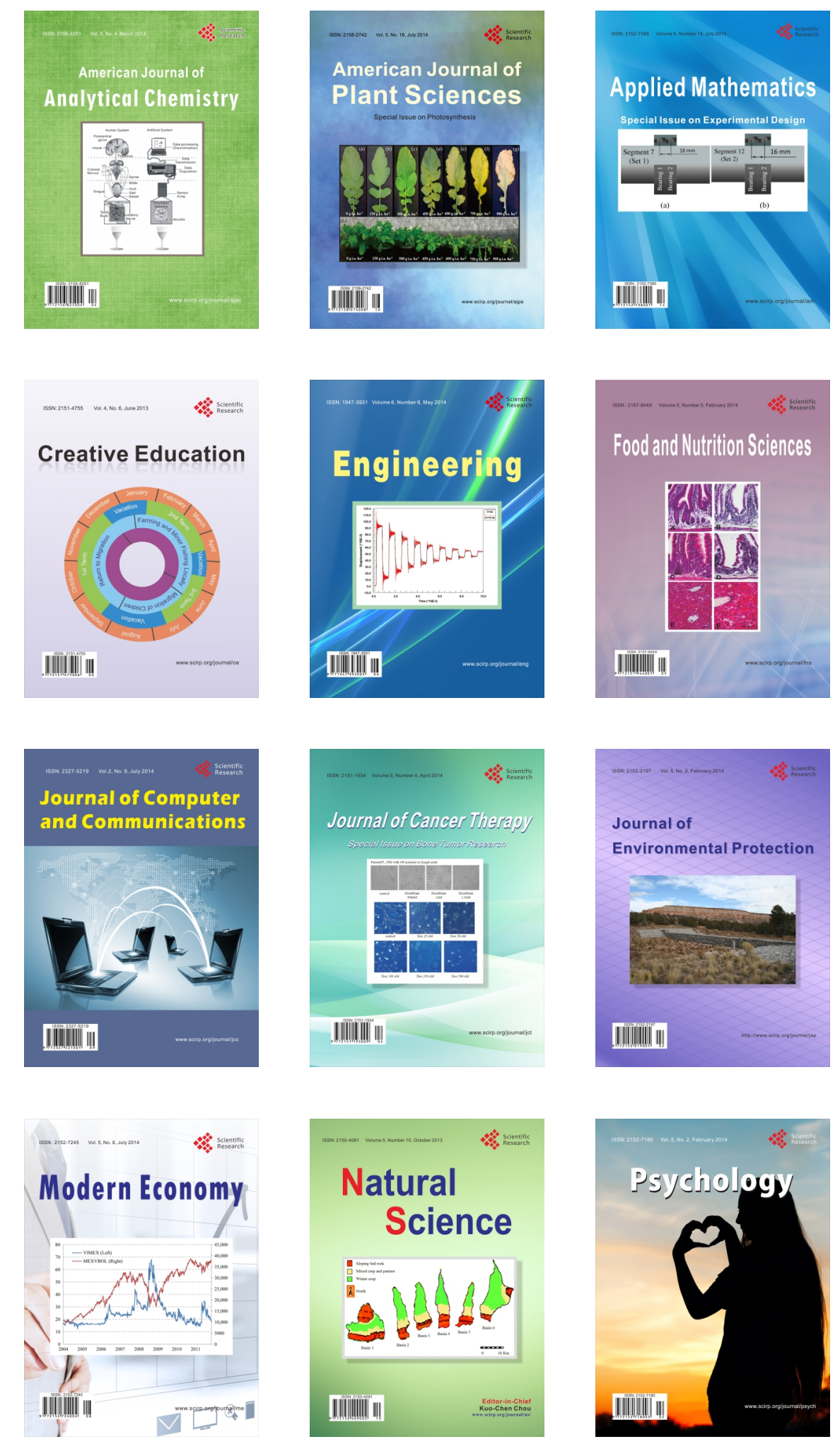